\title{
Relatório Final da XV Reunião Anual de Pesquisa Aplicada em Doença de Chagas e da III Reunião Anual de Pesquisa Aplicada em Leishmanioses
}

No ano de 1999 de comemoração dos 90 anos da descoberta da doença de Chagas, os temas básicos escolhidos para a reunião foram:

1. Desafios da doença de Chagas em centros urbanos e

\section{Controle da leishmaniose no Brasil}

O número de participantes foi superior aos anos anteriores: 279, incluindo principalmente pesquisadores das universidades e órgãos governamentais envolvidos nas estratégias de controle de ambas as endemias, estudantes de pós-graduação, além de estudantes de graduação. Estiveram presentes participantes de todo o Brasil: Pará, Piauí, Rio Grande do Norte, Pernambuco, Ceará, Bahia, Minas Gerais, Rio de Janeiro, São Paulo, Mato Grosso, Goiás, Distrito Federal, Paraná, Santa Catarina, Rio Grande do Sul, representando as diversas universidades ou institutos de pesquisa (Universidade de São Paulo, UNIFESP, UNICAMP, Universidade Estadual de Londrina, além das Universidades Federais de todos os estados citados. Contamos com a participação das várias unidades da FIOCRUZ: Instituto de Pesquisa Gonçalo Moniz, René Rachou, Oswaldo Cruz e Hospital Evandro Chagas. Representando a comunidade internacional estiveram presentes um pesquisador chefe do Setor de Doenças Tropicais e pesquisadores de vários institutos de pesquisa da Argentina, da Universidade de Buenos Aires, do Equador e da Universidade de Filadélfia.

Duas características enfatizadas e que tiveram um resultado acima do esperado foram: 1. a participação de pesquisadores de instituições acadêmicas e governamentais na definição de estratégias para o controle da doença de Chagas e da leishmaniose no Brasil (oficinas número quatro e sete), para abordar o controle pós-terapêutico da doença de Chagas (oficina número dois), a atenção médica para doença de Chagas em centros urbanos (oficina um), para conhecer a real situação da co-infecção HIVLeishmania e da infecção por Trypanosoma rangeli no Brasil; 2. a reunião de pesquisadores das áreas de imunologia, parasitologia, biologia, patologia, clínica, epidemiologia para propor soluções frente a questões específicas tanto nas oficinas de trabalho como nas mesas redondas.

Segue-se um resumo das principais atividades no Congresso:

1. Oficinas de trabalho, reunindo mais de 180 participantes, com um núcleo de 7 a 20 pesquisadores que, junto com o moderador e 0 presidente, traçaram previamente o roteiro, os conteúdos a serem apresentados para provocarem a discussão. Uma nova característica foi a abertura das oficinas para que jovens e participantes menos experientes pudessem não só ter acesso às questões principais do tema em foco mas também verificar como foram encaminhadas. Os nomes dos palestrantes e coordenadores, bem como do roteiro das sete oficinas estão apresentados no programa no início do livro de resumos. O resultado final deste trabalho está demonstrado no Anexol.

2. Sete mesas-redondas ocorreram, sendo três na área de epidemiologia e controle (Transmissão da doença de Chagas: como estamos?), com uma visão crítica da situação atual; vetores da doença de Chagas, com proposição de alternativas para o controle de triatomíneos como veículos na transmissão da doença de Chagas; e uma sobre Controle das ações de saúde em nível municipal na leishmaniose. Duas mesas redondas versaram sobre interação hospedeiroparasito na leishmaniose e na doença de Chagas, discutindo o papel das cepas e do hospedeiro, com uma proposta de pesquisadores brasileiros para uniformizar a classificação de Trypanosoma cruzi e novas ferramentas moleculares para o estudo de tropismo de clones de parasitos; duas mesas de clínica e terapêutica, uma sobre o diagnóstico e controle pós-terapêutico da leishmaniose, tema de interesse nacional na vigência da expansão do calazar no Brasil sem uma reação prática e acessível de referência,

Recebido em 17/1/2000. 
além de avanços em cardiopatia e enteropatia chagásica.

3. A sessão de posters contou com 83 apresentações, assim distribuídas:

a. Aspectos clínico-epidemiológicos da doença de Chagas I: 12 ;

b. Aspectos clínico-epidemiológicos da doença de Chagas II: 9;

c. Epidemiologia da doença de Chagas (vigilância de vetores): 7;

d. Aspectos clínico-epidemiológicos da leishmaniose: 11;

e. Aspectos clínicos e terapêuticos da leishmaniose: 14;

f. Diagnóstico parasitológico e terapêutica da doença de Chagas: 9;

g. Diagnóstico imunológico da doença de Chagas e da leishmaniose: 8

h. Imunologia e patologia da doença de Chagas: 7;

i. Imunologia e Patologia da leishmaniose: 6 .

Os trabalhos foram divididos em duas fases: uma frente ao poster, de discussão dos relatores com os expositores e uma de discussão com 0 grupo de participantes que tinham assuntos em comum em nove salas.

4. Foram premiados o melhor trabalho em doença de Chagas e o melhor trabalho em leishmaniose, tendo concorrido participantes que apresentaram o trabalho completo à comissão julgadora, que trabalhou em duas etapas: a primeira escolhendo dois finalistas e a segunda, selecionando o vencedor após a apresentação e discussão dos trabalhos. A comissão julgadora foi assim constituída: Presidente: Erney Plessman Camargo; Participantes: Maria Irma Seixas Duarte, Aluízio Prata, Maria Aparecida Shikanai Yasuda e Zilton de Andrade.

5. Foi oferecido um curso pré-congresso, que reuniu mais de 40 participantes, entre os quais estudantes de graduação e de pós-graduação stricto e lato sensu e funcionários da Fundação Nacional de Saúde, contando com o apoio de professores da Faculdade de Medicina de Uberlândia, da Faculdade de Medicina da Universidade Federal de Goiás, da Universidade de São Paulo e do Instituto Oswaldo Cruz, sob a coordenação de Virmondes Rodrigues Júnior e da Maria Aparecida Shikanai Yasuda.

6. A reunião contou com 4 conferências:

A doença de Chagas 90 anos após a sua descoberta: Prof. Aluízio Prata;

Leishmaniasis: global overview on public health aspects and control: Dr. Philippe Desjeux;

Desafios da doença de Chagas em centros urbanos: Dr. João Carlos Pinto Dias;

Perspectivas na pesquisa básica e aplicada na doença de Chagas e na leishmaniose no próximo século: Dr. José Rodrigues Coura.

7. Finalmente, um dos pontos altos da reunião foi a plenária, nesta reunião contemplada com um maior tempo para que todos os participantes pudessem participar da discussão e da aprovação dos relatórios das oficinas de trabalho e das recomendações para o próximo congresso, a ser realizado no ano 2000 , sob a coordenação dos Prof. Zilton Andrade e Sônia Andrade.

Devo ressaltar que a experiência de vários pesquisadores representando grupos nacionais e internacionais consolidados foi vital para o estímulo e engajamento de novos estudantes e que a experiência de reunir pesquisadores de diversas áreas do conhecimento em torno de perguntas concretas com uma pauta de discussão já elaborada foi reconhecida como ideal para o encaminhamento de propostas de trabalho, espírito este, que, segundo participantes, norteou a criação da reunião de pesquisa básica de doença de Chagas e que foi retomado nesta reunião.

São Paulo, 29 de novembro de 1999

\author{
Maria Aparecida Shikanai Yasuda \\ Coordenadora
}




\section{RELATÓRIOS DAS OFICINAS DE TRABALHO}

\section{“ATENÇÃO MÉDICA A CANDIDATOS A DOADORES DE SANGUE EM CENTROS URBANOS: INSERÇÃO NO SISTEMA DE SAÚDE (PROVAS SOROLÓGICAS POSITIVAS E INCONCLUSIVAS PARA DOENÇA DE CHAGAS)"}

\author{
Presidente: Hélio Moraes de Souza \\ Moderadora: Eliane Dias Gontijo
}

Participantes: Alejandro H. Moreno, Amaro Nunes Duarte, Ana M. Bonametti Cybele Kalid Santos; Daniel Bonomi, Eduardo Hage Carmo, Eliana Furtado Moreira, Eros Antônio de Almeida, Jamiro Wanderley, Maria da Glória Melo, Maria Virgínia Fernandes Camilo, Marta H. Lopes, Sérgio de A. Nishioka, Sheila Soares, Silvana Marques de Araújo, e Silvano Wendel

Relatores: Eliane Gontijo, Ana M. Bonametti, Marta H. Lopes e Silvano Wendel

\section{METODOLOGIA}

No período da manhã os participantes discutiram vários tópicos relacionados à dimensão do problema e ao manejo das pessoas com sorologias reativas para doença de Chagas, incluindo os métodos diagnósticos e os encaminhamentos mais adequados para esses indivíduos.

Identificados os problemas, o período da tarde foi reservado para elaboração de propostas direcionadas para dois temas principais:

1) atendimento do candidato a doador nos bancos de sangue

2) Identificação do nível de atendimento e manejo clínico-laboratorial das pessoas soropositivas ou com sorologia inconclusiva encaminhadas pelos bancos de sangue.

Estima-se que sejam colhidas cerca de 2.800 .000 bolsas de sangue por ano no Brasil, com $60 \%$ no setor público e $40 \%$ no setor privado. Desse total de doações, dados mais recentes mostram que $1 \%$ dos casos apresentam sorologia não-negativa para doença de Chagas. Considerando a média de 1,2 doações anuais por indivíduo, pode-se, estimar que, a cada ano, 25.000 candidatos a doador da hemorrede pública e privada do país apresentem reações reativas. Estes resultados obtidos após repetição na mesma amostra, usando-se dois testes com princípios diferentes, apresentam, com certeza, uma grande taxa de resultados falso-positivos.
As estimativas são de que 3.750 a $5.000(10 \%$ a $15 \%$ ) são positivos verdadeiros e 20.000 a $21.250(75 \%$ a $80 \%)$ apresentam resultados inconclusivos. Do total de pessoas com resultados sorológicos inconclusivos, somente 400 (2\% a 3\%) são confirmados como positivos verdadeiros, enquanto que a grande maioria (19.600 a 20.850), permanecem sem confirmação diagnóstica e representam o grande dilema quanto à abordagem clínica a ser seguida. Mesmo considerando que a grande maioria destes casos seja na realidade, falsa reatividade sorológica, alguma resposta deve ser dada a este contingente de pessoas.

Como tentativa e proposta de diminuir o número de casos inespecíficos, e objetivando não sobrecarregar o sistema de saúde o grupo sugeriu as seguintes abordagens:

a) Estimular os doadores de repetição (fidelizados) que representam cerca de $30 \%$ a $40 \%$ do total de doadores de sangue no país. O ideal é que se atinja de $80 \%$ a $90 \%$ dos casos.

b) Detectar as áreas geográficas que tenham maiores prevalências de doadores infectados e não fomentar a coleta de sangue nessas regiões.

c) Incentivar as indústrias produtoras de kits comerciais no sentido de desenvolver testes mais específicos, sem detrimento de suas sensibilidades

d) Garantir a repetição dos testes sorológicos dos doadores inconclusivos (o grupo de 19.600 a 20.850) numa segunda amostra, que deveria ser responsabilidade dos laboratórios de referência (LACEN), que encaminharia os positivos para o sistema de saúde.

e) Promover o desenvolvimento de testes confirmatórios para doença de Chagas, à semelhança do que ocorre com HIV, HVC e HTLVI/II.

f) Manter a rede de laboratórios de saúde pública de referência com as atribuições de garantir o controle de qualidade e formar agentes multiplicadores para execução do diagnóstico sorológico em suas respectivas regiões.

Em relação ao atendimento dos candidatos a doador de sangue no sistema de saúde identificou-se que a maioria dos centros de atenção ao paciente chagásico representados nessa oficina pertencem a hospitais universitários e não têm interligação adequada com a Rede 
Básica de Saúde do local. Poucas experiências tem sido desenvolvidas na implantação da assistência ao chagásico em uma rede descentralizada, como ocorre na Bahia, onde foram implantadas 8 unidades, e em Pernambuco, no município de Igarassu.

Tal situação tem gerado uma sobrecarga aos serviços universitários, o que tem desvirtuado seu papel de centros de referência. Por outro lado, a imensa maioria de chagásicos é atendida na rede de assistência sem uma oferta organizada ou um melhor nível de qualificação e de recursos diagnósticos e terapêuticos.

A proposta básica discutida no grupo, busca a inserção da assistência ao paciente chagásico na rede descentralizada do SUS, tendo como eixo a municipalização, e a reestruturação do papel dos centros de referência, enquanto centros de capacitação, assessoria, monitoramento, assistência de maior complexidade e pesquisa, atuando em apoio às unidades básicas.

Para garantir $o$ atendimento integral e com equidade de todos os soropositivos foram encaminhadas as seguintes propostas:

1. Os candidatos a doador de sangue com resultados sorológicos não-negativos para doença de Chagas ou seja, aqueles com sorologias comprovadamente positivas ou inconclusivas, podem ser adequadamente atendidos nos serviços de atenção primária à saúde, por equipe de profissionais capacitados, articulada com os Programas de PACS/PSF, desde que os profissionais que atuam nesses serviços estejam informados sobre as características clínicas e laboratoriais da doença de Chagas e instruídos por manuais de padronização de condutas de fácil leitura e interpretação. Este nível seria a porta de entrada dos pacientes chagásicos, incluindo os candidatos à doadores encaminhados pela rede hemoterápica com provas sorológicas não negativas. Deve-se identificar a rede de apoio para realização das provas sorológicas e outros exames .

2. Os participantes se dispõem a colaborar na elaboração de um manual onde poderiam constar informações relevantes para o manejo desses pacientes, tais como:

- Não é necessária a repetição sorológica nos pacientes com resultados sorológicos comprovadamente positivos por duas técnicas diferentes, preferencialmente, quantitativas. As técnicas recomendadas são: hemaglutinação, imunofluorescência indireta e imunoenzimática (ELISA).

- Para as pessoas que não preencherem os critérios acima citados deverá ser coletada nova amostra para repetição dos exames sorológicos pelas mesmas técnicas.

- Os que permanecerem com resultados inconclusivos após a segunda coleta, deverão ser submetidas a duas novas colheitas, com intervalo de seis meses. Se essa duas novas amostras forem negativas a pessoa será considerada como nãochagásica e deverá receber alta do serviço. Os critérios de negatividade aprovados pelo grupo são: resultados negativos em duas amostras de sangue empregando-se três técnicas sorológicas diferentes, preferentemente quantitativas.

- As pessoas que permanecerem com resultados inconclusivos ou duvidosos após os procedimentos citados acima deverão entrar na rotina de atendimento dos pacientes da fase crônica da doença de Chagas, explicitada adiante.

- Os pacientes com sorologia positiva deverão ser acompanhados, na rede primária, por equipe preferentemente multidisciplinar, capaz de atender o chagásico em sua globalidade que transcende o enfoque tradicional biologicista. Para os pacientes assintomáticos, essa primeira avaliação deverá ser complementada somente com a solicitação de eletrocardiograma com D2 longo. Esses pacientes deverão ser reavaliados a intervalo mínimo de um ano e máximo de cinco e em cada reavaliação os mesmos procedimentos da primeira consulta deverão ser efetuados. Deverá ser garantido atendimento às intercorrências que se fizerem presentes neste intervalo de tempo.

- Para os pacientes sintomáticos a avaliação será completada com exame radiológico simples de tórax (com determinação do índice cárdio-torácico) no caso de sintomatologia sugestiva de comprometimento cardíaco e exame radiológico de esôfago e/ou enema opaco quando o paciente apresentar queixas compatíveis com comprometimento digestivo.

- Os pacientes com alguma alteração nos exames complementares citados poderão ser encaminhados para avaliação especializada, a critério do médico responsável. Após a 
definição do quadro e orientação terapêutica, os pacientes poderão retornar ao serviço de origem, desde que não seja necessário o acompanhamento contínuo pelo especialista.

- Os pacientes acompanhados nos serviços de atendimento primário poderão ser tratados especificamente, de acordo com as normas adotadas pelo Ministério da Saúde, desde que esses serviços disponham de profissional médico capacitado para tal procedimento.

- Atendimento de pacientes encaminhados pelos outros níveis, que requeiram procedimentos terapêuticos como implantação de marca-passo, cirurgias de megas, etc, ou procedimentos diagnósticos não disponíveis nos demais níveis deverá ser garantido no nível terciário de atenção.

3. Centros de Referência

Os participantes da oficina que atualmente exercem o atendimento especializado a doentes chagásicos, solicitam que o Ministério de Saúde do Brasil reconheça esses serviços como de referência para o desenvolvimento de atividades de pesquisa, elaboração de normas técnicas, manutenção de atividades assistenciais especializadas, assessoria técnica aos demais níveis, promoção e/ou execução das capacitações, monitoramento das demais unidades, em articulação com o Sistema de Vigilância Epidemiológica de cada Estado.

Para a implantação/implementação desta forma de organização propõe-se:

a. Identificação dos centros de referência existentes no país;

b. Elaboração de agenda da capacitações (manejo clínico, tratamento específico, leitura de ECG), com identificação e garantia de recursos financeiros e definição de atribuições em cada Estado, em articulação com as SES;

c. Elaboração de manual técnico sobre padronização de condutas: Manejo clínico do paciente chagásico;

d. Estruturação dos Centros de Referência para desenvolvimento de assessoria às demais unidades, definindo financiamento para ações de supervisão em conjunto com a SES; implantação de rede de informações (via internet) para assessoria, consultas, educação à distância; e. Financiamento da rede básica para aquisição de equipamentos e insumos;

f. Apoio governamental para a realização de reuniões com a participação dos coordenadores dos Centros de Referência a Gerência Técnica de Doença de Chagas e a Coordenação de Sangue e Hemoderivados do Ministério da Saúde.

\section{“CONTROLE PÓS-TERAPÊUTICO DA DOENCA DE CHAGAS. QUAIS AS TÉCNICAS A SEREM UTILIZADAS?"}

Presidente: Joaquim Romeu Cançado

Moderador: Alejandro O. Luquetti

Relatores: José Franco da Silveira e José Mauro Peralta

\section{INTRODUÇÃO}

Basicamente, a avaliação de cura de pacientes tratados especificamente, tanto na fase aguda como na crônica da infecção chagásica, tem sido feita por meio do xenodiagnóstico com 40 triatomíneos e das provas sorológicas convencionais (IFI, HAI e ELISA), pela técnica quantitativa.

A relativa baixa sensibilidade do exame parasitológico na fase crônica e a demora na alteração dos títulos de anticorpos, têm levado investigadores a conclusões precipitadas, motivadoras de confusão no espírito daqueles que prestam assistência a pacientes chagásicos.

Cabe-nos, portanto, especialistas que somos, tentar dirimir as dúvidas existentes, padronizando a metodologia da avaliação de cura, principalmente em função da aquisição de novos métodos de investigação.

A pergunta inicial que fazemos é a seguinte: haveria métodos laboratoriais mais específicos e mais sensíveis que os já citados? Seus resultados denunciariam mais precocemente, ou antecipariam o resultado do tratamento avaliado pelos métodos convencionais?

Constam da literatura alguns trabalhos que procuram mostrar o significado deste ou daquele novo método de aferição de cura, cabendo-lhes, entretanto, uma maior avaliacao em estudos longitudinais, a partir do período pré-terapéutico. 


\section{OBJETIVO}

Estabelecer métodos para seleção e seguimento de pacientes após o tratamento etiológico, visando estabelecer critérios de cura, assim como verificar a viabilidade de estudos multicêntricos para avaliação de novos testes.

Em qualquer estudo de seguimento terapéutico devem ser realizados testes sorológicos e parasitológicos antes do início do tratamento.

\section{TESTES PARASITOLÓGICOS}

Os testes parasitologicos não devem ser utilizados como único exame diagnóstico da fase crônica da doença de Chagas, já que a parasitemia é freqüentemente intermitente, causa de resultados negativos.

Nos casos de sorologia duvidosa, os exames parasitológicos podem ser utilizados como métodos alternativos na busca diagnóstica e só tem valor quando positivos.

A reação em cadeia da polimerase ( $P C R$ ) é mais sensível e proporciona resultados mais rapidamente que a hemocultura e o xenodiagnóstico. Entretanto para a sua validação, a especificidade deve ser de $100 \%$, empregando amostras de indivíduos de áreas não endêmicas para Tripanosomíase Americana.

Acresce dizer que a execução dos métodos parasitológicos clássicos não deve ser interrompida, devido, entre outras razoes, à necessidade de isolamento de parasitos para seu posterior estudo.

O seguimento dos indivíduos submetidos a tratamento etiológico deve ser realizado por testes sorológicos e parasitológicos, sendo que o critério de cura atualmente vigente exige negativação de ambos os exames.

Recomenda-se a realização de testes parasitológicos antes do início do tratamento, para avaliar a parasitemia, embora resultado negativo não invalide o início do tratamento. Preconiza-se a realização de três exames prévios, com prazos de semanas a meses entre um e outro.

Há necessidade de padronização do PCR visando à uniformidade de resultados entre diferentes laboratórios. As condições básicas sugeridas são: sangue coletado com EDTA $(0,2 \mathrm{M})$-guanidina $(6 \mathrm{M})$; volume de sangue a ser coletado: em adulto, $10 \mathrm{ml}$, em criança, $5 \mathrm{ml}$ e em recém nascidos, $250 \mu$ l. Deve-se empregar iniciadores (primers) derivados da região constante do kDNA, recomendando-se a padronização de nomes, de acordo com a seqüência a seguir discriminada (Octavio, preencher, 535, 536?).

Cada amostra deve ser analisada em duplicata. A presença de inibidores da PCR deve ser investigada utilizando-se iniciadores direcionados para a betaglobina humana. Deve ser utilizada a enzima Taq polimerase adequada somente em elevadas temperaturas. Recomenda-se hibridização com utilização de sondas .... (similares) específicas.

\section{TESTES SOROLÓGICOS}

O diagnóstico sorológico da infecção chagásica está bem estabelecido, podendo ser empregadas diversas técnicas convencionais (IFI, HAI, ELISA) com essa finalidade .

Vários "kits" comerciais tem sido avaliados demonstrando alto grau de eficiência. Para a reprodutibilidade de resultados, considerou-se importante que os laboratórios que realizam diagnóstico sorológico tenham programas de controle de qualidade e obedeçam as regras de boas práticas de laboratório. Os resultados na sorologia convencional, para seguimento de pacientes tratados, devem ser expressados em título para uma correta avaliação evolutiva.

Foi considerada a necessidade de avaliação e desenvolvimento de marcadores sorológicos que possam servir como preditores de cura pósterapêutica uma vez que a sorologia convencional só se torna negativa após alguns lustros .

A lise mediada por complemento, com formas tripomastigotas vivas, tem demonstrado ser uma técnica eficiente; entretanto, devido a sua complexidade e baixa reprodutibilidade, tornase necessário a avaliação e desenvolvimento de novas técnicas empregando, talvez, antígenos da forma tripomastigota tanto no desenvolvimento de testes sorológicos convencionais como não convencionais .

Estudos multicêntricos foram propostos empregando grupos de pacientes não tratados previamente, que deverão ser avaliados clinicamente e por exames laboratoriais para a detecção de parasitos e anticorpos anti-T.cruzi por testes sorológicos convencionais, com seguimento após o tratamento a cada seis meses, nos dois primeiros anos, e depois 
anualmente, com avaliação pelas técnicas convencionais bem como por novas técnicas que vem sendo desenvolvidas em diversos laboratórios de pesquisa.

\section{"MARCADORES DE EVOLUÇÃO DAS FORMAS CLÍNICAS DA DOĚNÇA DE CHAGAS. COMO PROSSEGUIR?"}

Participantes: Vanize Macedo, Ricardo Ribeiro dos Santos, José Borges-Pereira, Anis Rassi Jr, Antonio R.L. Teixeira, Barbara Ianni, Claude Pirmez, Luiz Fernando Junqueira, Manoel Otávio Costa Rocha, Marcus Vinícius Simões, Rodrigo Correa, Sergio Sales Xavier e Tania C. Araujo Jorge

\section{CONSIDERAÇÕES GERAIS}

As formas clínicas da doença de Chagas são expressões dos diferentes resultados do processo de interação homem-Trypanosoma cruzi. A freqüência destas formas clínicas varia de acordo com as características regionais e com a metodologia de investigação empregada.

Os estudos clínicos-epidemiológicos seccionais e/ou longitudinais realizados, até o momento, configuraram as principais manifestações clínicas destas formas clínicas, e o conhecimento existente sobre a morbidade nos permite caracterizar uma ampla variação na evolução das formas clínicas, que vai da inalterabilidade por toda a vida à progressão continuada determinando à morte do indivíduo.

Os avanços alcançados no controle da transmissão vetorial é um fator determinante da redução do número de chagásicos. Contudo, é preciso avançar no controle da doença existente entre os milhões de infectados, Neste contexto emerge a questão que motiva esta oficina: a identificação de marcadores que nos indiquem os caminhos da evolução da doença, conduzindo-nos às possíveis medidas terapêuticas que possam modificar a história natural em benefício dos milhões de chagásicos existentes.

O grupo acredita que a abordagem clínica da doença de Chagas tem sido satisfatoriamente realizada com definições e padronizações como as sugeridas por peritos reunidos pelo $\mathrm{CNPq}$ em 1974, embora acredite que os resultados obtidos com a aplicação de métodos como a eletrocardiografia dinâmica e a ecocardiografia avaliando prognósticos possam contribuir para futuras modificações, as quais poderão, além de uniformizar a abordagem em torno dos marcadores, ampliar a análise comparativa dos resultados de diferentes grupos de estudos.

\section{MARCADORES DE EVOLUÇÃO PARA AS FORMAS DETERMINADAS}

O grupo concluiu que, até o momento, não se conhece marcador capaz de indicar quais pacientes evoluirão da forma indeterminada para as formas cardíaca, digestiva, mista ou outras. Contudo, as discussões em torno dos resultados de pesquisas realizadas em animais e humanos indicaram possíveis estudos a serem desenvolvidos:

1. Estabelecer linhagens/clones de células T humanas respondedoras a antígenos do T. cruzi e do tecido cardíaco molecularmente definidos

2. Ampliar os estudos sobre os níveis de citocinas em função das diferentes formas clínicas, particularmente de IL10, gama interferon e TGF beta

3. Em estudos longitudinais, avaliar a importância de glutation-peroxidase, CPK e Troponina nas diferentes formas clínicas

4. Estimular as pesquisas em torno de marcadores genéticos que possam estratificar populações ou grupos de indivíduos mais ou menos susceptíveis ao processo de infecção e ao desenvolvimento da doença.

\section{MARCADORES DE EVOLUÇÃO DAS FORMAS DETERMINADAS}

Os estudos realizados, até o momento, com casuísticas selecionadas ou probabilísticas consolidaram alguns marcadores do processo evolutivo das formas clínicas da doença de Chagas, principalmente aqueles determinados pela extensão do acometimento miocárdico, representados por alterações eletrocardiográficas como Bloqueio completo do ramo direito associado ao hemibloqueio anterior esquerdo, zona eletricamente inativa, taquicardia ventricular não-sustentada ao Holter e as extra-sístoles ventricular complexas polimórficas, e ecocardiográficas pela presença de disfunção ventricular esquerda de grau moderado ou severo, caracterizada por fração de ejeção inferior a $40 \%$. 
O grupo considera promissores, como possíveis marcadores precoces da doença, os resultados apresentados sobre alterações eletrocardiográficas mutáveis, as alterações da função sistólica e diastólica regional ao dopller tissular, sobre a denervação autonômica e os distúrbios da perfusão miocárdica evidenciados pela cintigrafia tomográfica e sobre a avaliação da função autômica através de diversos testes existentes.

\section{"ESTRATÉGIAS PARA VIGILÂNCIA EPIDEMIOLÓGICA DA DOENÇA DE CHAGAS NA PERSPECTIVA DA DESCENTRALIZAÇÃO DAS AÇÕES"}

Participantes: Aluízio Prata, Antonio Carlos Silveira, João Carlos Pinto Dias, Liléia Diotaiuti, Dalva Marli Valerio Wanderley, David E. Gorla, Claudio R. Lazzari, Eduardo Nicolas Zerba, Carlos Mauricio F. Antunes, Fabio A. Porto, Bernardina V.M. Azevedo, Jesus Alberto B. Benitez, Rogério da Silva Gomes, Sebastião Aldo Valente, Ridelane V. Acioli, João Luis Carvalho, Segovia Maria Amália.

\section{INTRODUÇÃO}

A doença de Chagas hoje apresenta baixo nível de prioridade para o controle devido a pressão de outras patologias que passaram a fazer parte do cenário do controle das endemias no Brasil. Além da freqüente inaparência clínica da moléstias, os próprios avanços logrados no controle do vetor fizeram, naturalmente, baixar as densidades domiciliares dos mesmos, o que diminui o interesse pelo seu combate. Esta é uma das grandes dificuldades para monitorar estar áreas o que exige uma vigilância epidemiológica continua e permanente.

Acresce ainda que, passada a fase tradicional de ataque, faz-se necessária a progressiva inserção de toda a comunidade nos trabalhos de vigilância, o que tem fugido da rotina institucional, gerando dificuldades para a implementação. A despeito do controle da transmissão do Triatoma infestans (Ti) a nível domiciliar, a presença deste vetor ainda é verificada em vários municípios principalmente dos estados da Bahia, Tocantins, Rio Grande do Sul e Minas Gerais. Além disso outras espécies vetoras importantes da doença como o Triatoma brasiliensis no Nordeste, ao lado daquelas nativas que se domiciliam e que podem transmitir a doença ao homem, carecem maior atenção. A realidade da VE hoje aponta problemas na sua implantação, os PITs apresentam baixa produtividade, ela não está integrada a educação, está voltada apenas para barbeiro, pouco aberta para outras formas de transmissão e pouco sustentada pelo programa e pela pesquisa.

Assim como a continuidade e contiguidade constituem ingredientes históricos que permearam o êxito obtido nas ações contra o Ti, a vigilância entomológica deve estar inserida na vigilância epidemiológica ai incluída a doença nos seus vários mecanismos de transmissão e a atenção do indivíduo infectado, e ser permanente. Entende-se, neste contexto, que o processo de descentralização das ações de saúde constitui um movimento de avanço e aperfeiçoamento, sendo necessário um grande esforço para compatibilizar ações específicas como o controle de doenças transmitidas por vetores com as novas estruturas e estratégias. Um grande risco é o simples desmonte de Instituições clássicas e bem organizadas como a FNS sem deixar-se no lugar nada que possa atender a esses tipos de agravos. Neste sentido, compete aos gestores do SUS e aos responsáveis pela FNS elaborar em conjunto o processo de transição, de maneira responsável e racional, sob pena de deixar descoberta a população afetada.

Para que este processo de implantação da VE se inicie devem ser envolvidas as instâncias formais do SUS, por meios dos conselhos nacionais, estaduais e municipais de saúde, além de outras alternativas como o Programa de Saúde da Família (mobilizar e detectar caso), Consórcio Municipal de Saúde (união em torno de problemas comuns) e dos Agentes de Saúde (ação educativa mais ampla). A questão do financiamento dessas ações é fundamental para a sua sustentabilidade.

Atualmente, se coloca de forma mais organizada por meio de uma rubrica própria inserida na NOB-99 (Controle de Endemias) com repasse de recursos fundo a fundo, garantida por habilitação dos municípios.

Supõe-se então que deve ser mantida a referência técnica para os municípios executores, o que justifica a existência das instâncias regionais (distritos) e da coordenação Estadual. Tal formatação exige o trabalho de compatibilização desses distritos com as estruturas próprias de cada estado, a exemplo do que já ocorre em São Paulo com a SUCEN/SES. 


\section{DISCUSSÃO}

Estratégias para a inclusão do tema "Chagas" na pauta de prioridades para a vigilância epidemiológica. É preciso informar e deixar mais claro para as instâncias de decisão a transcendência do problema representado pela DC ainda hoje, apesar dos níveis de controle alcançados, especialmente no caso da transmissão vetorial por $T$. infestans.

Nesse sentido deve-se estabelecer que o principal horizonte operacional no momento é a vigilância epidemiológica, a ser exercida de forma descentralizada, ampliando-se o mais possível a sua base de operação, pela incorporação dos serviços locais e regionais de saúde.

De outra parte deve ser incorporada como um componente de um sistema mais amplo de vigilância epidemiológica e ambiental, nos três níveis de governo, a ser desenvolvida de forma permanente e continuada sob pena de ser perder todo o investimento feito até aqui, com risco concreto de retrocesso da atual situação epidemiológica. Para tanto são fundamentais as discussões e esclarecimento do problema juntamente com os Estados (CONASS) e representações pertinentes dos municípios (CONASEMS) bem como a inclusão formal do tema na formulação do VIGISUS.

Em especial se recomenda total apoio e imediato desenvolvimento de inquérito sorológico nacional que proverá as bases epidemiológicas concretas para a adequada avaliação do problema e incremento da vigilância.

Propõe-se ainda discutir este tema ao ensejo da atual avaliação que vários Estados brasileiros estão fazendo para a "Certificação da interrupção da transmissão por Triatoma infestans".

Uma das maiores dificuldades para a incorporação de estados e municípios na vigilância da doença de Chagas em particular e, de resto, das doenças transmitidas por vetores (DTV) tem sido a falta de espertícia nestes níveis de gestão, o que inclusive limita a capacidade de absorção dessas ações. Isso determina a necessidade de que se desenvolvam estratégias e metodologias para a capacitação em larga escala de pessoal profissional e técnico.

Ademais, ao invés da criação de novas estruturas especializadas em estados e municípios julga-se recomendável considerar a possibilidade de transferência dos recursos já existentes da Fundação Nacional de Saúde (FNS) para as Secretarias Estaduais de Saúde, que viriam a constituir o embrião da Coordenações Estaduais de Vigilância Ambiental, o que concorda com a proposta contida na NOB-99, hoje apresentada pela FNS aos demais níveis de governo. Nessa proposta não se extingue a instância central da FNS, a qual competirão ações de normalização, acompanhamento e avaliação, assegurando a articulação das ações a nível nacional e internacional. Da mesma forma, se mantêm os níveis distritais de coordenação, referência técnica, supervisão e capacitação da instâncias municipais, como ocorre em São Paulo, tendo ainda, quando necessário condições de exercer atividades de ponta em caráter supletivo. Nesta perspectiva o modelo prevê que esses distritos sejam incorporados e sintonizados com as instâncias regionais das respectivas secretarias de estado de saúde, subordinadas a estas na conformidade da normativa SUS.

Estratégias para integrar os serviços locais de saúde nas ações de vigilância da doença de Chagas. A rede local deve estar preparada para identificar o triatomíneo, identificar o caso e dar o primeiro atendimento ao indivíduo infectado. Estas ações serão respaldadas pela instância regional, a qual caberá suplementar. Já existe uma experiência no estado do Pará onde a FNS está inserida na Secretaria da Saúde, reforçando o modelo. A promoção de um centro de referência regional poderá ser viabilizado com recursos do VIGISUS.

O nível central da FNS deve ser fortalecido no seu corpo técnico (recursos humanos) e no aporte financeiro (orçamento) pois a ele caberá o papel de síntese, orientação técnica e supervisão.

Os Conselhos Municipais de Saúde deverão ser estimulados pelos níveis regionais para promover a discussão das ações de vigilância da doença de Chagas a serem desenvolvidas e acompanhar a adequada alocação dos recursos no programa, como forma de garantir a continuidade das ações e o nível de compromisso pactuado, inclusive entre municípios vizinhos. Em particular, esta estratégias objetiva também prevenir o risco de câmbios de prioridades eventualmente devidas a mudanças políticas ao nível periférico.

Viabilidade de manutenção e necessidade de revisão das ações de vigilância no novo contexto de operação descentralizada. Esse item já está contemplado no modelo proposto, devendo salientar-se que a questão do orçamento é 
fundamental para que estados e municípios possam levar adiante as atividades necessárias. Um ponto chave nesse contexto corresponde as ações de contínua supervisão e acompanhamento epidemiológico, a serem assumidas pelo estado. Neste contexto, é importante que se determinem as normas para investigação de casos, com elaboração de protocolo sistematizado e introdução de "ficha de investigação de casos".

Necessidade de investigação (pesquisa aplicada) para uma maior eficácia e efetividade da vigilância, em especial para os mecanismos "secundários" de transmissão

- Desenvolver teste rápido para diagnóstico da fase recente da doença, que possa também atender os casos de transmissão congênitas.

- Estudo multicêntrico para determinar o risco de transmissão da doença de Chagas congênita no país

- Desenvolver um modelo para companhamento do processo de implantação e execução da vigilância epidemiológica.

- Métodos de detecção de vetor em áreas de baixa densidade, como por exemplo a utilização de sensores capazes de competir com refúgios naturais de triatomíneos.

- Investigar a competência vetorial de domiciliação das espécies secundárias, inclusive em termos de predição.

- Induz pesquisas na área de educação e mobilização comunitária.

- Desenvolver estudos do efeito do clima no comportamento de espécies triatomínicas em diferentes regiões do Brasil.

- Desenvolvimento de novos inseticidas para o controle químico de vetores secundários.

- Reproduzir estudos da eficácia de uso de inseticidas no controle das espécies triatomínicas.

- Desenvolvimento de produtos com ação desalojante e avaliar a indicação do seu uso na pesquisa triatomínica.

Situações especiais e temas específicos. Vários episódios de transmissão humana da doença de Chagas têm sido descritos e relacionados a diferentes espécies de triatomíneos. Recentemente, a ocorrência de Panstrogylus megistus no sul do Pará pode indicar a introdução passiva da espécie na região, revelando a falta de vigilância a dispersão dos triatomíneos para novas áreas.
A partir de 1994 foi concebido um modelo especial de VE para esta região, em que, a partir da detecção do novo caso, geralmente via Programa de Controle da Malária ou Serviço de Hemoterapia, procede-se a investigação epidemiológica com vista a elucidar o mecanismo de transmissão. Apesar do modelo ser factível sua implantação realmente só foi realizada nos estados do Pará e Amapá. Recomenda-se a sua ampliação para toda a Região, centralizando os esforços na busca de novos casos, o que seria obtido por meio da capacitação dos servidores que já trabalham na região, numa perspectiva de otimização dos recursos já existentes, para a identificação do T. cruzi e de triatomíneos.

Recomenda-se ainda a inclusão da Região Amazônica no novo inquérito sorológico que está sendo planejado para determinação da prevalência da doença de Chagas no Brasil.

\section{"INFECÇÃO POR TRYPANOSOMA RANGELP'}

Presidente: José Rodrigues Coura

Moderador: Alvaro José Romanha

Participantes: Marta M.G. Teixeira, Edmundo Carlos Grisard, Luís Eduardo Ramirez, Mário Steindel

Debatedoras: Maria Inês Machado, Cláudia de Abreu Fonseca

\section{CONSIDERAÇÕES/CONSTATAÇÕES}

Chamamos a atenção da comunidade científica para a existência do Trypanosoma rangeli de maneira sobreposta à área de distribuição do T. cruzi nas Américas Central e do Sul e especialmente no Brasil. O T. rangeli compartilha os mesmos reservatórios e vetores com o T. cruzi, já tendo sido assinaladas infecções mistas em reservatórios e vetores, bem como infecções humanas na região da Amazônia Brasileira.

A existência de Didelphis albiventris (gambá), Echimys dasythrix (roedor), Panstrongylus megistus e Rhodnius brethesi naturalmente infectados pelo T. rangeli no Brasil, bem como a existência de $R$. neglectus no Triângulo Mineiro e $R$. domesticus em Santa Catarina, ainda que não comprovadas suas infecções naturais, mas comprovadas as suas capacidades de transmissão experimental, mostram um cenário que amplia as possibilidades 
de achados de casos humanos de infecção pelo T. rangeli.

Detecção do T. rangeli. Em humanos e reservatórios animais:

- Hemocultura em meio ágar-sangue+LIT (NNN + LIT).

- Xenodiagnóstico utilizando espécies do gênero Rhodnius, devendo-se utilizar preferencialmente a espécie local. Em humanos deve-se realizar o xenodiagnóstico artificial e não o natural devido a resposta alérgica usualmente desencadeada pela saliva do inseto.

- Os exames de sangue a fresco ou esfregaços corados tem poucas chances de encontrar 0 parasita devido aos níveis muito baixos de parasitemia no homem e um pouco maiores, porém ainda baixos nos reservatórios animais, mesmo na fase aguda da infecção. Uma vez encontrado o parasita no esfregaço corado é possível diferenciar o $T$. rangeli do $T$. cruzi. O T. rangeli é mais longo que o T. cruzi, apresenta um cinetoplasto pequeno, puntual e subterminal, membrana ondulante mais pregueada e o núcleo pequeno e arredondado.

- O microhematócrito pode ser utilizado com boa sensibilidade em infecções recentes em humanos e na detecção parasitológica em reservatórios,

- Sorologia:Testes de imunofluorescência indireta e Elisa convencionais apresentam reatividade cruzada com o T. cruzi. Entretanto, métodos sorológicos que utilizem antígenos específicos de $T$. cruzi ou $T$. rangeli poderão ser utilizados na diferenciação dos parasitas.

- PCR: Ainda não se encontra disponível a PCR para a detecção de $T$. rangeli em sangue.

Em triatomíneos:

- Exame a fresco ou em esfregaços corados pelo método de Giemsa da saliva, da hemolinfa e do conteúdo das glândulas salivares.

- O exame das fezes não permite a identificação do $T$. rangeli, devido ao grande polimorfismo do parasita, podendo ser facilmente confundido com o T. cruzi.

- Detecção via PCR em amostras de fezes coletadas em papel de filtro.

Diferenciação específica entre T. cruzi e T. rangeli. Em humanos e reservatórios animais. A diferenciação específica entre as duas espécies ainda é baseada na necessidade de isolamento das amostras. Uma vez isolada a cepa do parasita (por hemocultura, inoculação em animais, xenocultura, etc...) deve-se utilizar técnicas biológicas, bioquímicas, imunológicas ou moleculares apropriadas e/ou disponíveis. Em triatomíneos a diferenciação de formas presentes nas fezes deve igualmente ser realizada utilizando-se técnicas biológicas, bioquímicas, imunológicas ou moleculares apropriadas e/ou disponíveis.

\section{RECOMENDAÇÕES}

1. Considerar a possibilidade de infecções únicas pelo T. rangeli e/ou mistas $T$. rangeli / T. cruzi tanto em reservatórios animais, insetos vetores e em humanos.

2. As análises por diferentes marcadores moleculares e genéticos mostram uma variabilidade entre as cepas de T. rangeliocorrendo nas Américas, entretanto permitindo reuni-los em grupos distintos. Por isso recomenda-se cautela na aplicação e na interpretação dos resultados obtidos pelas técnicas moleculares utilizadas em $T$. rangeli.

3. A posição sistemática do T. rangelié controversa e necessita ser discutida à luz das novas informações obtidas através das modernas técnicas moleculares, observando-se a correlação com os parâmetros biológicos e a procedência das amostras.

\section{“CO-INFECÇÃO LEISHMANIA/HIV"}

Participantes: Phillipe Desjeux, Ana Lúcia Rabello, Roberto Badaró, Valdiléia Gonçalves Veloso dos Santos. Giovanini Coelho, Alda Maria da Cruz, Fernando Tobias Silveira, Hiro Goto, Kleber Giovanni Luz, Marcelo Orsini, Márcia Hueb, Tânia Tozzeto Mendonça, Valdir Sabbaga Amato, Walkyria Pereira Pinto

Relatores: Alda Maria da Cruz, Fernando Tobias Silveira, Márcia Hueb, Ana Rabello

\section{CONSIDERAÇÕES GERAIS}

A co-infecção Leishmania/HIV é considerada pela Organização Mundial da Saúde como doença emergente e ameaçadora em vários países. A experiência mundial, principalmente européia, evidencia aumento importante do número de casos de co-infecção nesta década, levando a modificações no perfil epidemiológico 
e na apresentação e evolução clínica da leishmnaiose visceral naqueles países.

No Brasil, número crescente de casos de coinfecção vem sendo registrado em12 unidades da federação, abrangendo tanto a forma clínica tegumentar quanto a visceral. Entretanto, falta avaliação sistematizada da ocorrência real do coinfecção Leishmania/HIV e de sua implicação clínica e repercussão epidemiológica no país. A sistematização se faz necessária para a adequação dos critérios indicadores de suspeita clínica de co-infecção á realidade brasileira (Organização Mundial da Saúde, 1998; Badaró, 1997).

As recentes alterações nos perfis epidemiológicos identificados pelos Programas de Controle de DST/AIDS e de Leishmaniose no Brasil, como a interiorização e a pauperização da infecção pelo HIV, simultâneas à urbanização das leishmanioses, apontam para a possível expansão das populações em risco de adquirir as duas infecções.

Identifica-se neste momento, a necessidade de disseminação de informação com o objetivo de alertar os profissionais de intensificação da rede de notificações de co-infecção Leishmanial HIV no país e do aprimoramento do suporte diagnóstico da leishmaniose em conjunto com os serviços de referência para HIV/AIDS.

\section{RECOMENDAÇÕES}

Criação de uma Comissão de Estudos sobre a Co-infecção Leishmania/HIV, com participação de membros da comunidade científica e dos Programas de Controle de DST/AIDS, Leishmaniose Tegumentar e Leishmaniose Visceral do Ministério da Saúde. A primeira reunião da Comissão fica agendada para a ocasião do Congresso da Sociedade Brasileira de Medicina Tropical a se realizar em São Luís - Maranhão, e fevereiro de 2000. Ficarão a cargo da Comissão a organização e a coordenação das seguintes atividades:

1. Divulgação dos conhecimentos existentes sobre a co-infecção Leishmania/HIV pelos Programas e Coordenações de DST/AIDS e Leishmanioses, através de seus Boletins Epidemiológicos e outros meios de informação cabíveis;

2. Estabelecimento de uma rede de vigilância (centros-sentinela) de co-infecção Leishmanial HIV nos estados endêmicos para a leishmaniose, utilizando o sistema de serviços de referência para HIV do Programa DST/AIDS em colaboração com os serviços de referência para leishmanioses do país integrando a rede mundial de vigilância estabelecida pela Organização Mundial da Saúde.

\section{"ESTRATÉGIAS PARA O CONTROLE DA LEISHMANIOSE VISCERAL NO BRASIL: VETOR, CÃO, HOMEM"}

Presidente/Moderador: Carlos Henrique Neri Costa

Relator: Marco Tulio A. García-Zapata

Secretário: Gustavo A. Sierra Romero

Foi elaborado um relatório minucioso, que servirá como documento memória, base para as diversas reuniões de trabalho a serem realizadas junto aos diversos órgãos oficiais federais, estaduais e municipais (gestores, gerenciadores ou executores) do "Programa de Controle, Diagnóstico e Tratamento da Leishmaniose Visceral (Calazar)".

O que consta aqui é o resumo sucinto e dinâmico sobre os aspectos considerados mais polêmicos e de destaque que foram detectados no desenvolvimento e debate na Oficina de Trabalho "Estratégias para o Controle da Leishmaniose Visceral no Brasil: Vetor, Cão, Homem", e que foram aprovados na Reunião Plenária sobre a "Discussão dos Relatórios das Oficinas de Trabalho", no dia 07/11/99.

No desenvolvimento da reunião foram abordados e discutidos diferentes aspectos que partiram da descrição da situação atual da Leishmaniose Visceral Americana (LVA) no Brasil e contaram com a exposição de trabalhos técnicos e científicos sobre cada um dos fatores que de alguma maneira participam do cortejo epidemiológico e de controle da LVA.

Evidências constatadas (marco conceitual). $\mathrm{Na}$ abordagem expositiva e discussão dos temas, foram identificadas as seguintes evidências:

a) No ciclo epidemiológico de transmissão da leishmaniose visceral americana, o homem seria um importante reservatório ou fonte de infecção da $L(L)$ chagasi.

b) Até o momento, a magnitude da população humana que está infectada e assintomática não tem recebido a devida atenção no contexto da transmissão da LVA e do seu controle. 
c) A distribuição espacial da doença revela a possível existência de múltiplos fatores, potencialmente importantes na transmissão da doença, tais como a "ruralização do ambiente urbano e favelização", migração das populações humanas e desorganização na ocupação do espaço.

d) Há necessidade de mais evidências sobre o verdadeiro papel do cão como reservatório competente ou fonte de infecção da $L(L)$ chagasi na transmissão humana da LVA ${ }^{1}$.

e) Existem evidências sobre a diversidade genética das populações de Lu. longipalpis ${ }^{2}$ que teria influência sobre a sua eficiência como vetor, e também sobre a capacidade de dispersão e ocupação de novos ecótopos, o que poderia determinar mudanças nas estratégias de controle.

f) Faltam estudos consistentes sobre a influência dos diversos fatores ecológicos na dinâmica da transmissão da LVA, tais como os fatores sócio-econômicos, o saneamento básico, etc.

Problemática existente. Desconhecem-se os fatores que estão determinando o aparecimento cíclico (decenal) dos surtos epidêmicos. A identificação destes fatores esclareceria muitas das lacunas existentes no conhecimento atual e permitiria uma montagem racional de estratégias de controle viáveis e de baixo custo.

a) O sistema de controle vigente, em uso pelos órgãos oficiais, é baseado no modelo de intervenção integrada sobre os fatores envolvidos na epidemiologia da LVA porem carece de evidências técnico-científicas sólidas sobre a efetividade de cada um dessas ações de controle.

b) Existem diversos problemas operacionais na execução das ações preconizadas.

c) Existe uma tendência ao aumento indiscriminado no tratamento de cães infectados em áreas endémicas. d) Não existem indicadores ${ }^{3}$ viáveis e sobretudo confiáveis para orientar decisões relacionadas ao momento ideal e à qualidade de intervenção a ser aplicada, para: (i) Prevenir o aparecimento dos surtos (ii) Diminuir a incidência da LVA nas áreas endêmicas; (iii) Avaliar o impacto das ações.

e) Não estão estabelecidos os métodos que reconheçam a diversidade dos padrões de transmissão (urbano/rural), que seriam de especial importância diante da iminente descentralização das ações de saúde.

Recomendações/Sugestões. Avaliar ou reavaliar as ações de controle preconizadas pelo programa vigente, nos seguintes aspectos:

a) Medir o custo-beneficio e o custo-efetividade de cada uma das medidas de controle.

b) Implementar o estudo de medidas alternativas, possíveis e viáveis, frente à evidência da diversidade de fatores envolvidos no processo epidemiológico, incluídas aqui as vacinas para seres humanos e para cães.

Priorizar, em caráter de urgência, a revisão do "Manual de Controle, Diagnóstico e Tratamento da Leishmaniose Visceral (Calazar) - Normas Técnicas" ". Nesta revisão devem estar especificadas e descriminadas as ações próprias para o âmbito rural e para o urbano.

A Rede Nacional de Laboratórios de Saúde Pública (COLAB / CENEPI) deve participar de forma harmônica e integrada junto ao Sistema Nacional de Vigilância Epidemiológica ${ }^{5}$.

Estimular e apoiar projetos de pesquisa científica que visem aprofundar questões relativas com o estudo do homem como fonte infecção da $L$. (L) chagasi.

Estimular e apoiar projetos de pesquisa científica que visem estudar o cão como reservatório competente [e capaz], assim como

\footnotetext{
${ }^{1}$ Trabalhos recentes revelam muitas controvérsias neste sentido, como o referido por JBF Vieira na Revista da Sociedade Brasileira de Medicina Tropical 32 (Supl. II): 64-66, 1999, ou como o trabalho de O Courtenay, que mostra a pouca importância do cão na transmissão humana, publicado na Revista da Sociedade Brasileira de Medicina Tropical 32 (Supl. II): 101, 1999 (ambos apresentados na III Reunião de Pesquisa Aplicada em Leishmanioses).

${ }^{2}$ Shaw JJ.- Leishmaniose visceral no Brasil: O controle vetorial. Revista da Sociedade Brasileira de Medicina Tropical 32 (Supl. II): 98 , 1999 (apresentado na III Reunião de Pesquisa Aplicada em Leishmanioses).

${ }^{3}$ Parâmetros imunobiológicos, físicos, químicos, climáticos ou de outra natureza, necessários para avaliação de um evento de tal maneira a predefinir, em tempo, o curso de um determinado ciclo epidemiológico, assim como, a sua potencial alteração em benefício desse sistema.

${ }^{4} \mathrm{Em}$ vigência. Devido sobretudo, ao reconhecimento de evidências que questionam a real efetividade das ações de controle atualmente preconizadas.

${ }^{5}$ A RNLSP, deveria ser uma unidade do Ministério de Saúde, autônoma administrativamente, porém, com interfaces nos diferentes setores do Sistema, em benefício do controle e/ou erradicação das doenças endêmicas de interesse sanitário do país.
} 
avalia o real impacto da eliminação de cães no controle da LVA.

Não estimular o tratamento de cães infectados na áreas endêmicas de LVA, como medida de saúde pública.
Para que estas propostas sejam viabilizadas sugere-se que a FNS organize tão breve quanto possível, oficinas de trabalho para discutir analítica e criticamente as questões prioritárias, assim como buscar redefinir as ações relativas ao controle desta doença. 